\title{
NÍVEIS DE ENERGIA METABOLIZÁVEL PÓS PICO PARA POEDEIRAS LEVES CRIADAS EM GALPÕES ABERTOS DURANTE O PERÍODO DE INVERNO
}

\author{
(Energy levels metabolizable of the white laying hen rearing in the open shelter) \\ Marcos Rosniecek, Aline Schneider, Cleverson de Souza, Vladimir de Oliveira, \\ Clóvis Eliseu Gewehr ${ }^{1}$ \\ ${ }^{1}$ Correspondência: clovis.gewehr@udesc.br
}

\begin{abstract}
RESUMO: O efeito de níveis de energia metabolizável após o pico de postura foi avaliado em poedeiras leves criadas em galpão aberto durante o período de inverno na região Sul do Brasil utilizando 300 poedeiras $\mathrm{Hy}$-Line White com 40 semanas de idade, distribuídas em um delineamento inteiramente casualizado com 5 níveis de energia metabolizável (EM), por quilo na dieta $(2400,2600$, 2800,3000 e $3200 \mathrm{kcal} / \mathrm{kg}$ ) com seis repetições de dez aves. As aves foram submetidas as dietas experimentais durante um período de 40 dias sob temperatura média mínima de $7,31^{\circ} \mathrm{C}$ e a média máxima de $17,19^{\circ} \mathrm{C}$. Foi observado efeito $(\mathrm{P}<0,05)$ dos níveis de energia metabolizável da dieta sobre a produção de ovos, consumo de ração e conversão alimentar. A produção de ovos, a conversão alimentar e a massa de ovos apresentaram comportamento quadrático, sendo a maior produção obtida com $2561,51 \mathrm{kcal}$ de EM/kg, a menor conversão com 2434,34 kcal EM/kg e maior massa de ovos com $2548,52 \mathrm{kcal}$ de $E M / \mathrm{kg}$ de ração. Não foram observados efeitos ( $P>0,05)$ sobre o peso e densidade dos ovos e sobre o peso final das aves. Para poedeiras leves durante o período de inverno criadas em galpão aberto indica-se a utilização de 2561,51 kcal de EM/kg na dieta.
\end{abstract}

Palavras-chave: Galinhas, ovos, ração, postura

\begin{abstract}
The effect of metabolizable energy levels was evaluated in laying hens reared in open shed during the winter in southern Brazil using $300 \mathrm{Hy}$-Line White at 40 weeks of age distributed in a completely randomized design with 5 levels of metabolizable energy (ME) per $\mathrm{kg}$ in the diet $(2400$, $2600,2800,3000$ and $3200 \mathrm{kcal} / \mathrm{kg}$ ) with six replicates of ten birds each. The birds were subjected to treatment over a period of 40 days under $7.31^{\circ} \mathrm{C}$ mean minimum temperature and maximum average $17.19 \stackrel{\circ}{\circ}$. Effect was observed $(P<0.05)$, levels of metabolizable energy of diet on egg production, feed intake and feed conversion. Egg production, feed conversion, and egg mass showed a quadratic response, with the highest yield with $2561.51 \mathrm{kcal} / \mathrm{kg}$, the lowest conversion to $2434.34 \mathrm{kcal} / \mathrm{kg}$ and greater mass of eggs in $2548.52 \mathrm{kcal} \mathrm{ME} / \mathrm{kg}$ diet. No significant $(P>0.05)$ on the weight and density of the eggs and on the final body weight were observed. For light laying at 40 weeks of age, the winter climatic conditions of southern Brazil, indicates the use of $2561.51 \mathrm{kcal} / \mathrm{kg}$ in the diet.
\end{abstract}

Key Words: Hens, eggs, feed, posture 


\section{INTRODUÇÃO}

A interação entre consumo de ração, desempenho e temperatura ambiental são fatores a serem observados ao se formular dietas para poedeiras em diferentes regiões e estações do ano, visando uma combinação ideal entre estes (Donkoh e Atuahene, 1988).

As aves poedeiras são animais homeotérmicos que apresentam uma temperatura corporal de $41^{\circ} \mathrm{C}$. Quando submetidas a temperaturas que extrapolam sua zona de conforto tendem a regular sua homeotermia através do aumento ou redução no consumo de alimento, com intuito de manter a mesma ingestão de energia durante as diferentes estações do ano (Araújo e Peixoto, 2005). No entanto, esse mecanismo de controle não é totalmente eficiente, sendo que o consumo de alimento é regulado baseado no nível de energia na ração, além disso em temperaturas elevadas o mecanismo torna-se ainda mais deficiente (Leeson e Summers, 2005). Assim, quanto mais energia o alimento possuir, menor será o consumo pela ave, ocorrendo o inverso quando a energia é reduzida (Carioca et al., 2010).

Neste contexto quando as aves são criadas em temperaturas baixas como as que ocorrem no inverno do Sul do Brasil, tem sua exigência de energia de mantença aumentada, devido ao mecanismo de termo regulação, sendo necessária então mais energia dietética para suprir a exigência energética total destes animais (Ost e Peixoto, 2000). O trimestre composto pelos meses junho, julho e agosto caracteriza o período hibernal na região Sul do Brasil, onde as condições de tempo deste período são fortemente influenciadas por massas de ar vindas do continente Antártico, favorecendo a formação de geada e a queda acentuada da temperatura (Monteiro, 2001).
Além da baixa temperatura, outro fator importante que influencia a exigência dietética das aves é o período produtivo em que se encontram. Segundo Filho et al., (2006) o pico de postura vai da $26^{\text {a }}$ a $46^{\circ}$ semanas de idade, onde a produção de ovos ultrapassa o patamar de $90 \%$ sendo, provavelmente, o período em que a demanda de nutrientes se torna mais elevada, consequentemente, o nível de energia também se eleva, com intuito de manter a produção das aves.

Como no Brasil, a criação intensiva de aves é realizada em sua maioria em galpões abertos, com o objetivo de melhor aproveitar os recursos ambientais de cada região (Freitas et al., 2005), torna-se necessário realizar estudos que contemplem o ambiente em questão.

Com base no que foi descrito anteriormente, este trabalho objetivou avaliar o efeito de níveis de energia metabolizável na dieta de poedeiras leves de 40 semanas de idade criadas em galpões abertos durante o período de inverno na região Sul do Brasil sobre as características produtivas das aves.

\section{MATERIAL E MÉTODOS}

Este trabalho foi conduzido em um galpão experimental aberto, dotado com cortinas plásticas nas laterais, localizado na cidade de Lages - SC que apresenta latitude de $27^{\circ} 48^{\prime} 58^{\prime \prime} \mathrm{S}$, longitude de $50^{\circ} 19^{\prime} 34^{\prime \prime}$ e altitude de 884 metros. Essas coordenadas coincidem com a região considerada mais fria do Brasil.

Foram utilizadas 300 poedeiras leves com 40 semanas de idade, distribuídas em um delineamento inteiramente casualizado, com cinco tratamentos, seis repetições e 10 aves por unidade experimental. Os tratamentos a que as aves foram submetidas consistiram em cinco níveis de energia metabolizável (EM) na dieta, (2.400; 2.600; 2.800; 3.000 e 3.200 $\mathrm{kcal} / \mathrm{kg}$ ), durante um período de 40 dias, 
compreendido entre junho a agosto, onde a temperatura média mínima registrada no período foi de $7,31^{\circ} \mathrm{C}$ e a máxima $17,19^{\circ} \mathrm{C}$. As temperaturas extremas durante o período experimental obtidas na parte central do galpão foram $-1^{\circ} \mathrm{C}$ e $22^{\circ} \mathrm{C}$ para mínima e máxima, respectivamente.

As dietas experimentais (Tabela 1) foram formuladas a base de milho e farelo de soja, segundo as recomendações de Rostagno et al. (2005), com exceção dos níveis de energia metabolizável.

Tabela 1- Composição das dietas experimentais com base na energia metabolizável (kcal/kg).

\begin{tabular}{|c|c|c|c|c|c|}
\hline \multirow[b]{2}{*}{ Ingredientes } & \multicolumn{5}{|c|}{ EM (kcal/kg) } \\
\hline & 2.400 & 2.600 & 2.800 & 3.000 & 3.200 \\
\hline Milho & 46,34 & 58,65 & 60,30 & 55,54 & 50,12 \\
\hline Farelo de soja & 19,66 & 22,70 & 24,32 & 25,20 & 20,65 \\
\hline Farelo de trigo & 21,07 & 5,70 & - & - & - \\
\hline Óleo vegetal & - & - & 2,42 & 6,30 & 10,18 \\
\hline Calcário calc ́́tico & 9,93 & 9,95 & 9,96 & 9,96 & 9,96 \\
\hline Núcleo mineral e vitamínico* & 3,00 & 3,00 & 3,00 & 3,00 & 3,00 \\
\hline Total & 100 & 100 & 100 & 100 & 100 \\
\hline Proteína bruta (\%) & 16,00 & 16,00 & 16,00 & 16,00 & 16,00 \\
\hline Cálcio (\%) & 3,90 & 3,90 & 3,90 & 3,90 & 3,90 \\
\hline Fósforo(\%) & 0,34 & 0,34 & 0,34 & 0,34 & 0,34 \\
\hline \multicolumn{6}{|c|}{$\begin{array}{l}{ }^{*} \text { Formulado para atender as exigências de } \mathrm{P}, \mathrm{NaCl} \text {, microminerais e vitaminas } \\
\text { conforme recomendações de Rostagno et al. (2005). }\end{array}$} \\
\hline
\end{tabular}

As aves passaram por um período de duas semanas de adaptação às dietas anterior ao início do período de avaliação. Durante o período experimental foi utilizado programa de luz de 16h/dia (natural + artificial).

Foi avaliado consumo de ração (g/ave/dia), produção de ovos (\%ovos/ave/dia), conversão alimentar $(\mathrm{g} / \mathrm{g})$, peso de ovos $(\mathrm{g})$, massa de ovos (g), densidade dos ovos $\left(\mathrm{g} / \mathrm{cm}^{3}\right)$ e peso das aves ( $\mathrm{g}$ ). O peso e a densidade dos ovos foram avaliados através da média de todos os ovos produzidos nos últimos 3 dias do período experimental, sendo a densidade avaliada em baldes contendo solução de sal ( $\mathrm{Na} \mathrm{Cl})$, com densidades variando de 1,070 a $1,100 \mathrm{~g} / \mathrm{cm} 3 \mathrm{e}$ gradiente de 0,005 entre elas.

Os resultados das médias foram submetidos a análise de variância e regressão e as diferenças submetidas ao teste de Dunett utilizando o procedimento PROC GLM do software computacional SAS® (2003).

\section{RESULTADOS E DISCUSSÃO}

Ocorreu diferença $(P<0,05)$ entre os níveis $2.400,3.000$ e $3.200 \mathrm{kcal} / \mathrm{kg}$ de EM quando comparados ao controle $(2.800 \mathrm{kcal} / \mathrm{kg}$ ) para o consumo de ração (Consumo). As aves submetidas ao nível de $2.400 \mathrm{kcal} / \mathrm{kg}$ EM apresentaram maior consumo de ração, em contrapartida, aquelas que receberam dietas com níveis superiores, 3.000 e $3.200 \mathrm{Kcal} / \mathrm{kg}$ EM, tiveram menor consumo (Tabela 2). O consumo de ração é controlado pelo nível de energia da dieta (Leeson e Summers, 2005) e quando a energia da dieta é aumentada, o consumo tende a reduzir (Ost e Peixoto, 2000). Isto acontece devido ao fato da ave tentar manter um determinado consumo de energia diário, porém nem sempre este mecanismo ocorre de maneira correta, pois a influência da temperatura ambiental diminui ainda mais a eficiência da utilização da energia (Leeson e Summers, 2005; Carioca et al., 2010).

Tabela 2 - Desempenho zootécnico de poedeiras semipesadas submetidas a diferentes níveis de energia metabolizável sob temperaturas de inverno da Região Sul do Brasil. 


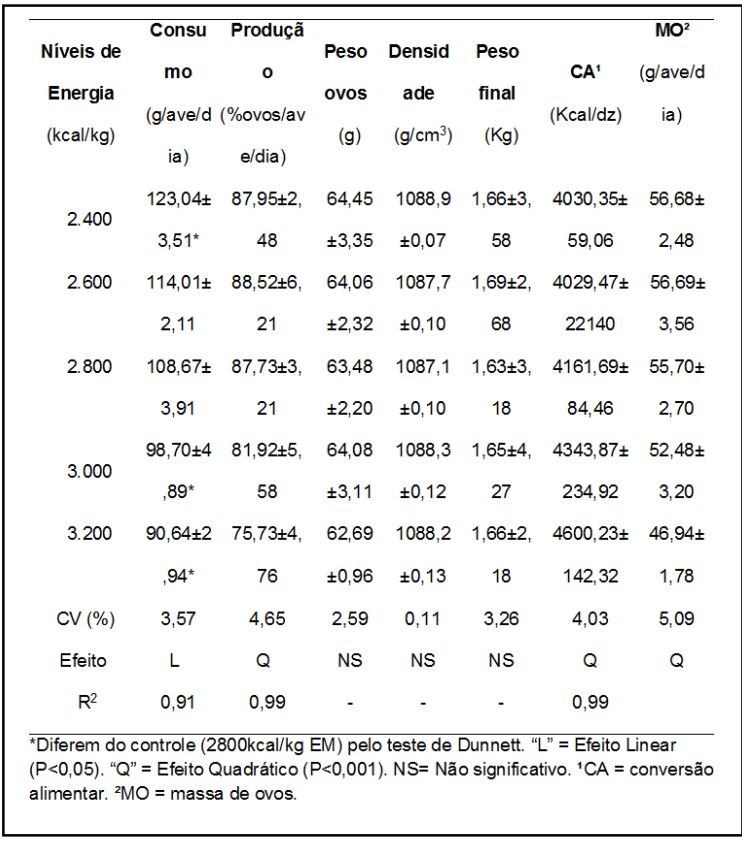

Trabalho realizado no inverno na região Sul do Brasil com poedeiras Isa Brown, indicou efeito linear no consumo de ração em ralação ao nível energético da ração, onde aves que ingeriram maiores níveis de energia na ração apresentam menor consumo (Ost e Peixoto, 2000). Entretanto, em outro estudo realizado por Xavier e Peixoto, (1997), com poedeiras Leghorn brancas nas mesmas condições climáticas e geográficas não observaram diferenças no consumo quando as aves receberam dietas com níveis variando entre 2.650 a $2.950 \mathrm{kcal}$ de EM. O mesmo foi observado por Araújo e Peixoto, (2005) em poedeiras Hisex Brown, sendo que estes autores também não encontraram diferenças no consumo das aves com dietas contendo níveis de energia entre 2.450 e $2.750 \mathrm{kcal}$ de EM.

A produção de ovos (Produção), a conversão alimentar (CA) e a massa de ovos (MO) apresentaram diferenças estatísticas $(P>0,05)$ e a análise de regressão indicou um comportamento quadrático (Figura 1) em relação aos níveis de EM da dieta. A maior produção de ovos foi obtida com o nível de $2.561,51 \mathrm{kcal} / \mathrm{kg}$ de EM na dieta em relação a conversão alimentar, o menor valor foi obtido quando as aves receberam 2.434,34 $\mathrm{kcal} / \mathrm{kg}$ de EM na dieta, já para a massa de ovos o maior valor foi obtido com o fornecimento de $2.548,52$. Efeito quadrático dos níveis de energia na produção de ovos é relatado por Xavier e Peixoto (1997), sendo que o resultado chegou próximo a 2.800 $\mathrm{kcal} / \mathrm{kg}$ de EM na dieta para obtenção de maior produção. O mesmo efeito foi observado por Araújo e Peixoto, (2005), no entanto os autores encontraram maior produção de ovos com o fornecimento de aproximadamente $2.615 \mathrm{kcal}$ de EM na dieta

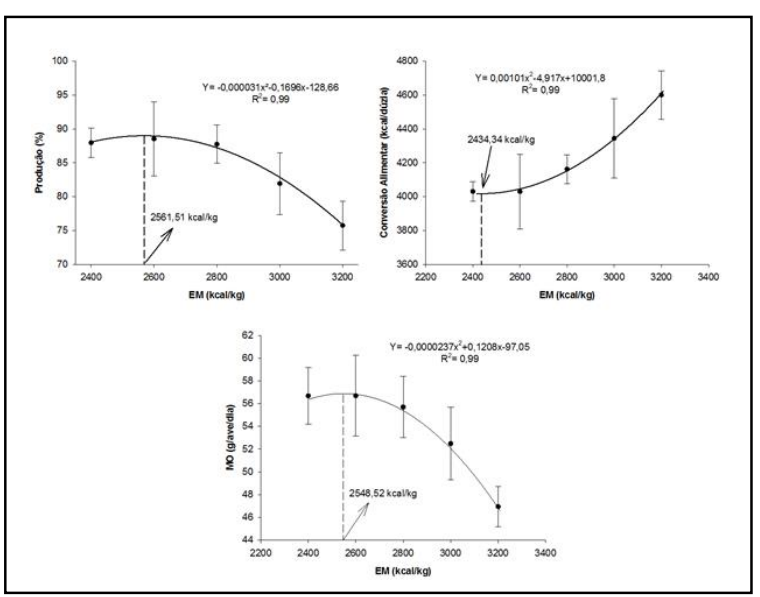

Figura 1: Resposta das variáveis produção de ovos, conversão alimentar e massa de ovos aos níveis de energia metabolizável na dieta de poedeiras de 40 semanas de idade criadas em galpão aberto nas condições de inverno do Sul do Brasil.

Com a elevação nos níveis de energia ocorre a redução da ingestão de alimentos (Leeson e Summers, 2005; Zhang et al., 2008). Sobre este aspecto, como as dietas são isonutritivas, instigase que pode ter ocorrido uma ingestão deficitária de proteína e aminoácidos, o que explica o comportamento dos dados de produção de ovos. Entretanto, a não influencia dos níveis de energia da dieta sobre a produção de ovos também é relatada na literatura, pois no trabalho de Ost e Peixoto, (2000), não foi observado 
efeito entre os níveis 2.650 e 2.950 $\mathrm{kcal} / \mathrm{kg}$ sobre a produção de ovos.

No tocante a conversão alimentar, efeitos similares foram encontrados por Costa et al., (2009), que observaram comportamento quadrático da conversão em relação aos níveis de energia na ração, sendo que estes autores indicam o valor de $2.830 \mathrm{kcal} / \mathrm{kg}$ de EM na dieta de poedeiras. Contudo, há trabalhos em que foram encontrados efeitos lineares dos níveis energéticos sobre a conversão alimentar, onde a conversão aumentou com o aumento dos níveis de energia na, sendo que os níveis utilizados variavam de 2.650 a $2.950 \mathrm{e}$ 2.450 a $2.750 \mathrm{kcal}$ de EM na dieta, respectivamente (Xavier e Peixoto, 1997; Ost e Peixoto, 2000; Araújo e Peixoto, 2005).

Embora o consumo de alimentos tenha sido inferior para aves que foram alimentadas com níveis superiores de $\mathrm{EM}$, a mesmas foram limitadas na produção de ovos, dessa forma ocorreu um aumento na conversão alimentar. Já as aves que consumiram menos energia tiveram, consequentemente, maior consumo de ração conseguindo compensar este aumento sobre a produção de ovos, resultando em uma menor conversão alimentar.

Os resultados encontrados para massa de ovos demonstram que as aves que consumiram 2.548, 52 kcal. $\mathrm{kg}$ de EM na dieta apresentaram uma maior massa de ovos. Isso se deve ao fato de que a massa de ovos é calculada pela razão da produção de ovos e peso dos ovos. Como a produção apresentou efeito quadrático e o peso do ovo não se alterou, a massa de ovos se comportou de maneira semelhante a produção de ovos. No entanto, Ost e Peixoto, (2000); Araújo e Peixoto, (2005) não observaram efeito dos níveis 2.650 a $2.950 \mathrm{kcal}$ de EM das dietas sobre a massa de ovos.
Para as demais variáveis, peso de ovo, densidade e peso final das aves não foram observados efeitos dos níveis de energia utilizados nas dietas. Estes resultados são corroborados com os encontrados por Araújo e Peixoto, (2005), que também não encontraram efeitos dos tratamentos sobre o peso do ovo e peso final das aves. Resultados contrários foram relatados por Ost e Peixoto, (2000); Xavier e Peixoto, (1997), os quais indicam efeitos da adição de EM na dieta sobre a densidade dos ovos, peso dos ovos e peso final das aves.

\section{CONCLUSÃO}

Para poedeiras leves de 40 semanas de idade criadas em galpões abertos nas condições climáticas de inverno da região sul do Brasil, indica-se a utilização de 2.561,51 kcal de EM/kg na dieta.

\section{REFERÊNCIAS}

Araújo, J.; and Peixoto, R. Níveis de energia metabolizável em rações para poedeiras de ovos marrons nas condições de inverno no extremo sul do Brasil. Arch. Zootec v.54, p.13-23, 2005.

Carioca, S.T.; Guimarães Cruz, F.G.; Matos, P.G.J.; et al. Influência dos níveis energéticos e protéicos em rações de poedeiras leves em manaus. Arch. Zootec. v.59, p.1-4, 2010.

Costa, F.G.P.; Costa, J.S. da; Goulart, C. de C.; et al. Metabolizable energy levels for semi-heavy laying hens at the second production cycle. Rev. Bras. Zootec. v.38, p.857-862, 2009.

Donkoh, a.; and Atuahene, C.C. Management of environmental temperature and rations for poultry production in the hot and humid tropics. Int. J. Biometeorol. v.32, p.247-253, 1988.

Filho, J.J.; Silva, J.H.V. da; Silva, E.L. da; et al. Exigência de lisina para poedeiras semipesadas durante o pico de postura. Rev. Bras. Zootec. v.35, p.1728-1734, 2006.

Freitas, H.J. De; Cotta, J.T.D.B.; Oliveira, A.I.G. de; et al. Avaliação de programas de iluminação sobre o desempenho zootécnico de poedeiras leves. Ciênc. agrotec. v.29, p.424-428, 2005. 
Leeson, S.; and Summers, J. Commercial poultry nutrition. 3rd ed. Guelph.

Monteiro, M.A. Caracterização climática do estado de Santa Catarina: uma abordagem dos principais sistemas atmosféricos que atuam durante o ano. Geosul v.16, p.69-78, 2001. 